\title{
Palliative Therapie des kolorektalen Karzinoms
}

\author{
C.-H. Köhne \\ Universitätsklinikum Carl Gustav Carus der Technischen Universität Dresden
}

Schlüsselwörter

Kolorektales Karzinom · Irinotecan - 5-Fluoruracil, Infusion · Oxaliplatin

\section{Zusammenfassung}

Die zytostatische Chemotherapie ist wesentlicher Bestandteil der palliativen Therapie von Patienten mit metastasiertem kolorektalen Karzinom. Gegenüber einer rein supportiven Behandlung verbessert eine auf 5-Fluorouracil (5-FU) basierende Chemotherapie die Lebensqualität und verlängert das Überleben der Patienten. 5FU-Dauerinfusion moduliert mit Folinsäure ist die beste Grundlage für die Kombination mit Irinotecan oder Oxaliplatin. Randomisierte Studien zum Einsatz von Irinotecan zeigten signifikante Vorteile im Hinblick auf die Remissionsrate, das progressionsfreie Überleben und auch die mediane Überlebenszeit. Randomisierte Studien zum Einsatz von Oxaliplatin zeigten ebenfalls höhere Remissionsraten und ein verlängertes progressionsfreies Überleben ohne eine verlängerte Überlebenszeit nachweisen zu können. Heutzutage sollten alle Patienten mit einer Kombinationschemotherapie behandelt werden und im Verlauf ihrer Erkrankung, soweit möglich, alle zur Verfügung stehenden Medikamente erhalten. Nur dadurch können mediane Überlebenszeiten von über 20 Monaten erreicht werden. Der Einsatz oraler Fluoropyrimidine statt einer 5-FU-Dauerinfusion in Kombination mit Irinotecan und Oxaliplatin ist viel versprechend, jedoch Gegenstand laufender Studien. Monoklonale Antikörper gegen den EGF-Rezeptor bzw. gegen VEGF haben ebenfalls viel versprechende Ergebnisse gezeigt und werden wahrscheinlich die Behandlungsmöglichkeiten in der Zukunft wesentlich verbessern.

\author{
Key Words \\ Colorectal cancer - Irinotecan - 5-Fluoruracil, infusional . \\ Oxaliplatin
}

\section{Summary}

Palliative Therapy of Colorectal Cancer

Systemic chemotherapy has a key role in the palliative treatment of patients with metastatic colorectal cancer. Compared to best supportive care, 5-fluorouracil (5-FU)based therapy prolongs survival and improves quality of life. 5-FU continuous infusion modulated by Leukovorin (LV) is the optimal basis for a combination therapy with irinotecan or oxaliplatin. Randomized trials investigating the role of irinotecan in combination with 5-FU/LV relative to 5-FU/LV alone demonstrated a significant improvement in the response rate, progression free survival and overall survival. Randomized studies using oxaliplatin/5-FU/LV vs. FU/LV alone resulted in a higher response rate and longer progression-free survival while the overall survival was not significantly different. Today, all patients should receive combination treatment in first line and should be offered all active compounds during the course of their disease. Hereby, median survival times of more than $\mathbf{2 0}$ months are achievable. The use of oral fluoropyrimidines as a substitute of infusional 5-FU in combination with irinotecan or oxaliplatin is promising and subject of clinical trials. Monoclonal antibodies directed against the EGF-receptor or against VEGF have demonstrated interesting results and may be a treatment option in the future.

\begin{tabular}{ll}
\hline KARGER & @ 2003 S. Karger GmbH, Freiburg \\
Fax +497614520714 & Accessible online at: \\
$\begin{array}{l}\text { E-mail Information@Karger.de } \\
\text { www.karger.com }\end{array}$ & www.karger.com/onk
\end{tabular}

Prof. Dr. med. Claus-Henning Köhne

Universitätsklinikum Carl Gustav Carus der Technischen Universität Dresden Medizinische Klinik und Poliklinik 1 Fetscherstraße 74, D-01307 Dresden Tel. +49 351 458-4780, Fax -5859

E-mail claus-henning.koehne@uniklinikum-dresden.de 


\section{Einleitung}

Seit fast 50 Jahren gilt 5-Fluoruoracil (5-FU) als die wichtigste Substanz in der palliativen Behandlung von Patienten mit einem metastasierten kolorektalen Karzinom [1]. Die Applikation als protrahierte Infusion über Tage oder Wochen sowie die biochemische Modulation insbesondere durch Folinsäure gelten als die wichtigsten Ergebnisse zahlreicher klinischer Studien der 1980er und 1990er-Jahre [2, 3]. Erfreulicherweise haben sich die Behandlungsmöglichkeiten durch Einführung oraler Fluorpyrimidine und insbesondere der Topoisomerase1-interaktiven Substanz Irinotecan sowie durch Einführung des Diaminocyclohexan-(DACH)-Oxaliplatin deutlich verbessert.

\section{Orale Fluoropyrimidine}

Orale Fluoropyrimidine geben Hoffnung auf eine patientenfreundlichere und nebenwirkungsärmere Therapie. Capecitabine und UFT (Uracil/Ftorafur) sind in großen randomisierten Studien gegenüber einem Bolus-5-FU-haltigen Regime getestet worden (Tab. 1) und zeigten ähnliche Remissionsraten, etwa gleich lange progressionsfreie Überlebenszeiten und keine Unterschiede in der medianen Überlebenszeit [4-7]. Die Toxizität der oralen Fluoropyrimidine bezüglich der gastrointestinalen und hämatologischen Nebenwirkungen war jedoch durchweg günstiger. Für Capecitabine ist ein Hand-FußSyndrom charakteristisch.

Capecitabine hat zumindest in einer Studie eine höhere Remissionsrate gegenüber der 5-FU-Bolus-Therapie gezeigt. Dieses Medikament scheint zur Zeit das am breitesten akzeptierte orale Fluoropyrimidin zu sein. Orale Fluoropyrimidine sind für alle diejenigen Patienten geeignet, bei denen keine Kombinationsbehandlung möglich erscheint. Da sich zunehmend die Kombinationschemotherapie durchsetzt, wird in der Zukunft interessant sein, welchen Stellenwert diese oralen Fluoropyrimidine in Kombination mit Irinotecan oder Oxaliplatin gegenüber der Kombination mit Dauerinfusion besitzen.

\section{Irinotecan}

Nachdem Irinotecan in der Zweitlinientherapie in zwei unabhängigen Studien entweder gegenüber «best supportive care» oder gegenüber einer 5-FU-Dauerinfusion eine signifikante Verlängerung der medianen Überlebenszeit bei 5-FU-vorbe-
Tab. 1. Orale fluoropyrimidinbasierte Prodrugs in der Erstlinientherapie (Phase-IIIStudien)

\begin{tabular}{lllll}
\hline & $\begin{array}{l}\text { Patienten, } \\
\mathrm{n}\end{array}$ & $\begin{array}{l}\text { Remissions- } \\
\text { rate, } \%\end{array}$ & $\begin{array}{l}\text { TTP/PFS, } \\
\text { Monate }\end{array}$ & $\begin{array}{l}\text { Medianes } \\
\text { Überleben, } \\
\text { Monate }\end{array}$ \\
\hline UFT/Folinsäure vs. & 190 & 11 & 3,4 & 12,2 \\
Mayo-/NCCTG-Regime [6] & 190 & 9 & 3,3 & 11,9 \\
UFT/Folinsäure vs. & 409 & 12 & $3,5^{*}$ & 12,4 \\
Mayo-/NCCTG-Regime [7] & 407 & 15 & $3,8^{*}$ & 13,4 \\
Capecitabin vs. & 301 & 19 & 5,2 & 13,2 \\
Mayo-/NCCTG-Regime [4] & 301 & 15 & 4,7 & 12,1 \\
Capecitabin vs. & 302 & $25^{* *}$ & 4,3 & 12,5 \\
Mayo-/NCCTG-Regime [5] & 303 & $16^{* *}$ & 4,7 & 13,3 \\
\hline
\end{tabular}

TTP: Mediane Zeit bis zur Tumorprogression, PFS: medianes progressionsfreies Überleben. $* \mathrm{p}=0,01 ; * \mathrm{p}=0,005$.
Tab. 2. Kombinationstherapie mit Irinotecan, Folinsäure und 5-Fluorouracil in der Erstlinientherapie (Phase-III-Studien)

\begin{tabular}{|c|c|c|c|c|c|c|c|}
\hline \multirow[t]{2}{*}{ Schema } & \multirow{2}{*}{$\begin{array}{l}\text { Patienten, } \\
\mathrm{n}\end{array}$} & \multicolumn{2}{|c|}{ Remissionsrate } & \multicolumn{2}{|l|}{ TTP } & \multicolumn{2}{|c|}{ Medianes Überleben } \\
\hline & & $\%$ & $\mathrm{p}$ & Monate & $\mathrm{p}$ & Monate & $\mathrm{p}$ \\
\hline AIO- oder de Gramont-Schema [9] vs. & 199 & 23 & \multirow{2}{*}{0,001} & 4,4 & \multirow{2}{*}{0,001} & 14,1 & \multirow{2}{*}{0,028} \\
\hline AIO/Irinotecan oder de Gramont/Irinotecan & 188 & 41 & & 6,7 & & 17,4 & \\
\hline ILF-Regime (Saltz-Regime) [10] vs. & 231 & 39 & \multirow{3}{*}{0,001} & 7,0 & \multirow{3}{*}{0,004} & 14,8 & \multirow{3}{*}{0,04} \\
\hline Mayo-/NCCTG-Regime [4] vs. & 226 & 21 & & 4,3 & & 12,6 & \\
\hline Irinotecan (Monotherapie) [5] & 226 & 18 & & 4,2 & & 12,0 & \\
\hline AIO (FU 2,6 g/m²) [11] vs. & 216 & 32 & \multirow{2}{*}{$<0,001$} & 6,4 & \multirow{2}{*}{$<0,0001$} & 16,9 & \multirow{2}{*}{0,028} \\
\hline $\mathrm{AIO}\left(\mathrm{FU} 2,0 \mathrm{~g} / \mathrm{m}^{2}\right) /$ Irinotecan & 214 & 54 & & 8,5 & & 20,1 & \\
\hline
\end{tabular}

TTP: Mediane Zeit bis zur Tumorprogression. 
Tab. 3. Kombinationstherapie mit Oxaliplatin, Folinsäure und 5-Fluorouracil in der Erstlinientherapie (Phase-III-Studien)

\begin{tabular}{|c|c|c|c|c|c|c|}
\hline \multirow[t]{2}{*}{ Schema } & \multirow{2}{*}{$\begin{array}{l}\text { Patienten, } \\
\mathrm{n}\end{array}$} & \multicolumn{2}{|c|}{ Remissionsrate } & \multicolumn{2}{|c|}{ TTP/PFS } & \multirow{2}{*}{$\begin{array}{l}\text { Medianes } \\
\text { Überleben, } \\
\text { Monate }\end{array}$} \\
\hline & & $\%$ & $\mathrm{p}$ & Monate & $\mathrm{p}$ & \\
\hline $\begin{array}{l}\text { de Gramont-Regime [13] vs. } \\
\text { FOLFOX }\end{array}$ & $\begin{array}{l}210 \\
210\end{array}$ & $\begin{array}{l}22 \\
51\end{array}$ & 0,0001 & $\begin{array}{l}6,2 \\
9,0\end{array}$ & 0,0003 & $\begin{array}{l}14,7 \\
16,2\end{array}$ \\
\hline $\begin{array}{l}\text { 5-FU/Folinsäure (chronomoduliert) [3] vs. } \\
\text { 5-FU/Folinsäure (chronomoduliert)/ Oxaliplatin [12] }\end{array}$ & $\begin{array}{l}100 \\
100\end{array}$ & $\begin{array}{l}16 \\
53\end{array}$ & 0,001 & $\begin{array}{l}6,1 \\
8,7\end{array}$ & 0,048 & $\begin{array}{l}19,9 \\
19,4\end{array}$ \\
\hline $\begin{array}{l}\text { Mayo-/NCCTG-Regime [4] vs. } \\
\text { 5-FU/Folinsäure/Oxaliplatin [14] }\end{array}$ & $\begin{array}{l}124 \\
114\end{array}$ & $\begin{array}{l}23 \\
49\end{array}$ & 0,0001 & $\begin{array}{l}5,3 \\
7,8\end{array}$ & 0,001 & $\begin{array}{l}16,1 \\
21,4\end{array}$ \\
\hline
\end{tabular}

TTP: Mediane Zeit bis zur Tumorprogression, PFS: medianes progressionsfreies Überleben.
Tab. 4. Oxaliplatin- oder irinotecanbasierte Kombinationstherapie mit 5-Fluorouracil/ Folinsäure in der Erstlinientherapie im randomisierten Vergleich (Phase-III-Studien)

\begin{tabular}{lllll}
\hline Schema & $\begin{array}{l}\text { Patienten, } \\
\mathrm{n}\end{array}$ & $\begin{array}{l}\text { Remissionsrate, } \\
\%\end{array}$ & $\begin{array}{l}\text { TTP, } \\
\text { Monate }\end{array}$ & $\begin{array}{l}\text { Medianes } \\
\text { Überleben, } \\
\text { Monate }\end{array}$ \\
\hline ILF-Regime [3] vs. & 264 & $29^{*}$ & $6,9^{* * *}$ & $14,1^{* *}$ \\
$\begin{array}{l}\text { FOLFOX [4] vs. } \\
\text { Irinotecan/Oxaliplatin [16] }\end{array}$ & 267 & $38^{*}$ & $8,8^{* * *}$ & $18,6^{* *}$ \\
Erstlinientherapie & 264 & $28^{*}$ & $6,7^{* * *}$ & $16,5^{* *}$ \\
FOLFIRI vs. & 109 & 56 & & \\
FOLFOX & 111 & 54 & 8,5 & 20,2 \\
«Cross-over» bei Tumorprogression & & & 8,1 & 21,5 \\
FOLFOX vs. & 81 & 14 & & \\
FOLFIRI [17] & 69 & 4 & 4,1 & - \\
\hline
\end{tabular}

TTP: Mediane Zeit bis zur Tumorprogression. * $\mathrm{p}=0,03 ;{ }^{* *} \mathrm{p}=0,002 ;{ }^{* * *} \mathrm{p}=0,0009$. handelten Patienten zeigen konnte [8, 9], gilt dieses Medikament als Therapie der Wahl für 5-FU-vorbehandelte Patienten. Die Einführung von Irinotecan in der Erstlinientherapie in Kombination mit 5-FU war daher der logische Schritt. Studienergebnisse legen nahe, dass die 5-FU-Dauerinfusion mit Irinotecan die optimale 5-FU-Applikation für eine Kombination ist. In 3 randomisierten Studien wurde eine 5-FU-Dauerinfusion moduliert mit Folinsäure mit oder ohne Irinotecan geprüft bzw. ein wöchentliches 5-FU-Bolusregime mit oder ohne Irinotecan getestet [9-11] (Tab. 2). Durch Irinotecan wurde in allen 3 Studien die Remissionrate signifikant verbessert und auch die progressionsfreie Überlebenszeit signifikant verlängert. In 2 Studien verlängerte Irinotecan signifikant die mediane Überlebenszeit der Patienten von 14,1 auf 17,4 bzw. von 12,6 auf 14,8 Monate. In der EORTC-Studie 40986 wurde die längste mediane Überlebenszeit einer 5-FU-Dauerinfusion mit Irinotecan (AIO-Schema) von über 20 Monaten beobachtet. Obwohl im Kontrollarm die mediane Überlebenszeit nur bei 16 Monaten lag, war dieser Unterschied statistisch nicht signifikant.

Erfreulicherweise war die Rate an therapieassoziierten Nebenwirkungen in allen Studien nicht wesentlich erhöht. In
Abhängigkeit vom Schema und der verwandten Irinotecandosis kommt es zu Alopezie in unter 5-20\% der Patienten bzw. zur schwerwiegenden Diarrhöe Grad III/IV bei etwa 20\% der Patienten. Durch geeignete Dosisreduktion bzw. konsequenten Einsatz supportiver Maßnahmen mit Loperamid bzw. Antibiotika - falls notwendig - gilt diese Therapie als sicher. Aufgrund der vorliegenden Therapieergebnisse ist insbesondere die 5-FU-Dauerinfusion mit Folinsäure und Irinotecan eine Referenz- oder Standardtherapie bei Patienten mit metastasiertem kolorektalen Karzinom.

\section{Oxaliplatin-basierte Kombinationstherapie}

Die Wirksamkeit einer Oxaliplatin-basierten 5-FU/Folinsäure-Kombination in der Erstlinientherapie wurde in 3 randomisierten Studien gegenüber 5-FU/Folinsäure geprüft [12-14] (Tab. 3). In allen Studien wurde durch Oxaliplatin eine signifikante Verbesserung der Remissionsrate und des progressionsfreien Überlebens erreicht. Obwohl die medianen Überlebenszeiten der Oxaliplatin-haltigen Therapie mit 16-20 Monaten recht hoch lagen, war dies statistisch nicht signifikant 
Tab. 5. Phase II- Studien mit Capecitabine (d1-14, qd21) und unterschiedlichen Gaben von Irinotecan

\begin{tabular}{llll}
\hline Autor & $\begin{array}{l}\text { Patienten } \\
\text { auswertbar, n }\end{array}$ & $\begin{array}{l}\text { Irinotec- } \\
\text { angabe }\end{array}$ & $\begin{array}{l}\text { Remissions- } \\
\text { rate, \% }\end{array}$ \\
\hline Kerr et al. [26] & 27 & alle 3 Wochen & 48 \\
Patt et al. [27] & 25 & alle 3 Wochen & 50 \\
Munoz et al. [28] & 42 & alle 3 Wochen & 45 \\
Dickson et al. [29] & 16 & wöchentlich & 38 \\
Bollina et al. [30] & 19 & wöchentlich & 32 \\
Tewes et al. [31] & 37 & wöchentlich & 38 \\
Grothey et al. [32] & 68 & Tag 1+8 & 43 \\
Cassata et al. [33] & 34 & Tag 1+8 & 71 \\
Park et al. [34] & 24 & Tag 1+8 & 50 \\
Gold et al. [35] & 29 & Tag 1+8 & 47 \\
Borner et al. [36] & 38 & wöchentlich & 29 \\
& 37 & alle 3 Wochen & 43 \\
\hline
\end{tabular}

unterschiedlich zum Kontrollarm. Die häufigsten Nebenwirkungen einer Oxaliplatin-haltigen Therapie sind neben einer Diarrhöe und hämatologischen Toxizität die für Oxaliplatin charakteristische kälteassoziierte Neurotoxizität.

\section{Kombinationen als Erstlinientherapie oder sequentielle Behandlung}

Der Stellenwert einer Zweitlinientherapie beim metastasierten kolorektalen Karzinom gilt als gesichert [8, 9]. Irinotecan kann das Überleben signifikant verlängern. In einer randomisierten Studie zur Zweitlinientherapie nach Irinotecan-Versagen war eine Kombination aus 5-FU-Dauerinfusion und Oxaliplatin einer alleinigen Behandlung mit Oxaliplatin oder einer alleinigen 5-FU-Dauerinfusion bezüglich der Remissionsrate und des progressionsfreien Überlebens überlegen, ohne einen Unterschied im medianen Gesamtüberleben zeigen zu können [15].

In 2 randomisierten Studien wurde Irinotecan in Kombination mit 5-FU gegenüber einer Kombination aus 5-FU und Oxaliplatin geprüft. In einer großen Studie, die in den Vereinigten Staaten durchgeführt wurde, zeigte sich, dass FOLFOX (5FU-Dauerinfusion/Folinsäure/Oxaliplatin) dem IFL-Regime (Irinotecan/5-FU als Bolus/Leukovorin) hinsichtlich der Rate der objektiven Remission in der Zeit bis zur Tumorprogression sowie des medianen Überlebens signifikant überlegen war [16] (Tab. 4). Die wahrscheinlichen Gründe liegen darin, dass im Kontrollarm (IFL) 5-FU als Bolus und nicht als kontinuierliche Infusion wie im FOLFOX-Regime gegeben wurde. Darüber hinaus stand für Patienten, die das IFL-Regime bekamen, Oxaliplatin nur innerhalb von Studien für die Zweitlinientherapie zur Verfügung, während Patienten, die zunächst FOLFOX erhielten, die Standardrezidivtherapie mit Irinotecan in hohem Prozentsatz erhalten konnten.

Die Therapieergebnisse waren nicht unterschiedlich, wenn FOLFIRI (Irinotecan, 5-fluorouracil infusion and leucovorin)

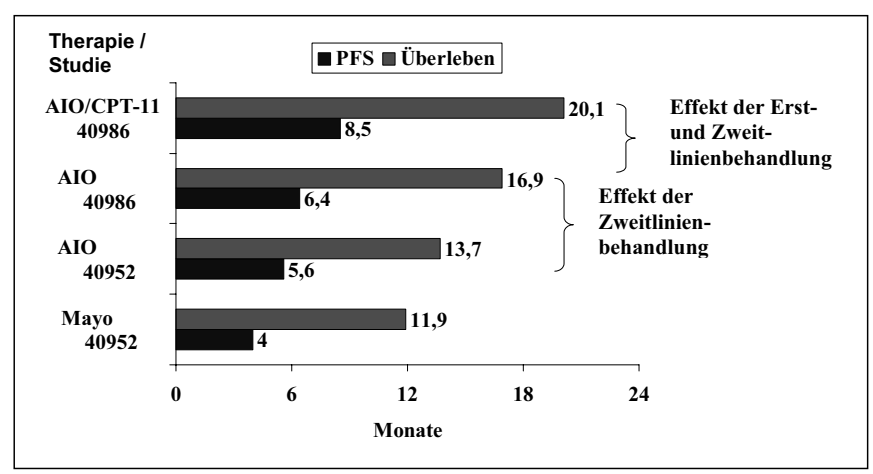

Abb. 1. Effekte von Erst- und Zweitlinientherapie auf die Überlebenszeit. PFS: Progressosfreies Überleben.

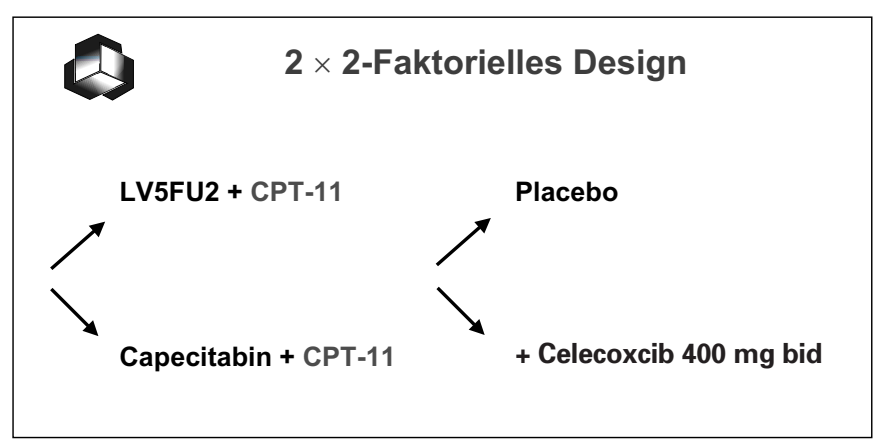

Abb. 2. EORTC-Studie 40015.

gefolgt von FOLFOX oder FOLFOX gefolgt von FOLFIRI in einem geplanten Cross-over-Design eingesetzt wurde [17]. Hier zeigte sich in beiden Therapieregimen eine über 50\%ige Remissionsrate und eine nahezu identische mediane progressionsfreie Überlebenszeit von über 8 Monaten. Durch den konsequenten sequentiellen Einsatz beider Therapieregime zeigten sich im medianen Überleben eine lange Überlebenszeit von 20 Monaten in beiden Therapiearmen. FOLFOX war in der Zweitlinientherapie mit einer etwas höheren Remissionsrate assoziiert, als dies mit FOLFIRI der Fall war, ohne dass dies allerdings statistische Signifikanz erreichte.

Die Effekte von Erst- und Zweitlinientherapie auf die Überlebenszeit lassen sich gut an den Erfahrungen der EORTCStudien 40952 [18] und 40986 [11] zeigen. Die Studie 40952 vergleicht das Mayo-Klinik-Schema mit dem AIO-Regime. Die Überlebenszeit war nicht statistisch signifikant unterschiedlich, jedoch die mediane progressionsfreie Zeit. Nur einige wenige Patienten erhielten in dieser Studie eine Zweitlinienbehandlung mit Irinotecan oder Oxaliplatin. Im Rahmen der EORTC-Studie 40986 wurde das AIO-Schema als Referenzbehandlung eingesetzt. In dieser Studie erhielten $65 \%$ der mit dem AIO-Schema behandelten Patienten eine Zweitlinientherapie (überwiegend Irinotecan). Dies wird wesentlich zu der medianen Überlebenszeit von fast 17 Monaten beigetragen haben. Falls die Patienten jedoch primär mit AIO plus Irinotecan behandelt wurden und dann $\mathrm{zu}$ fast $60 \%$ eine Zweitlinientherapie erhielten (2/3 Oxaliplatin, 1/3 Irinotecan) 
Tab. 6. EGF-

Rezeptor-Antikörper: Cetuximab

\begin{tabular}{|c|c|c|c|c|c|}
\hline & $\begin{array}{l}\text { Patienten, } \\
\mathrm{n}\end{array}$ & $\begin{array}{l}\text { Vorbehandlung/ } \\
\text { Studienphase }\end{array}$ & Dosis und Schema & $\begin{array}{l}\text { Remissionsrate, } \\
\%\end{array}$ & $\begin{array}{l}\text { PFS, } \\
\text { Monate }\end{array}$ \\
\hline Saltz et al. [37] & 121 & $\begin{array}{l}\text { Irinotecan/ } \\
\text { Phase II }\end{array}$ & Cetuximab + Irinotecan & 22,5 & \\
\hline Saltz et al. [38] & 57 & $\begin{array}{l}\text { Irinotecan/ } \\
\text { Phase II }\end{array}$ & Cetuximab & 10,5 & 1,7 \\
\hline Lutz et al. [39] & 21 & $\begin{array}{l}-/ \\
\text { Phase I }\end{array}$ & $\begin{array}{l}\text { Cetuximab }+ \\
\text { Irinotecan } 80 \mathrm{mg} / \mathrm{m}^{2}+\mathrm{AIO}\end{array}$ & 67 & \\
\hline Rosenberg et al. [40] & 29 & $\begin{array}{l}-/ \\
\text { Phase I }\end{array}$ & Cetuximab + IFL & 48 & \\
\hline Cunningham et al. [20] & $\begin{array}{l}218 \\
111\end{array}$ & $\begin{array}{l}\text { Irinotecan/ } \\
\text { Phase III }\end{array}$ & $\begin{array}{l}\text { Cetuximab } \\
\text { Cetuximab + Irinotecan }\end{array}$ & $\begin{array}{l}11^{*} \\
23^{*}\end{array}$ & $\begin{array}{l}1,5^{* *} \\
4,1^{* *}\end{array}$ \\
\hline
\end{tabular}

PFS: Medianes progressionsfreies Überleben. ${ }^{*} \mathrm{p}<0,01 ;{ }^{* *} \mathrm{p}<0,0001$

\begin{tabular}{|c|c|c|c|c|c|c|}
\hline & $\begin{array}{l}\text { Patienten, } \\
\mathrm{n}\end{array}$ & $\begin{array}{l}\text { Behandlung/ } \\
\text { Studienphase }\end{array}$ & Regime & $\begin{array}{l}\text { Überleben, } \\
\text { Monate }\end{array}$ & $\begin{array}{l}\text { Ansprechrate, } \\
\%\end{array}$ & $\begin{array}{l}\text { PFS, } \\
\text { Monate }\end{array}$ \\
\hline \multirow{3}{*}{$\begin{array}{l}\text { Bergsland et al. } \\
\text { [41] }\end{array}$} & 36 & \multirow{2}{*}{$\begin{array}{l}\text { Erstlinien- } \\
\text { therapie } \\
\text { randomisiert/ }\end{array}$} & \multirow{3}{*}{$\begin{array}{l}\text { FU/FA bolus wöchentlich } \\
\text { FU/FA bolus wöchentlich } \\
\text { rhuMab VEGF } 5 \mathrm{mg} / \mathrm{kg} \text {, q2w } \\
\text { FU/FA bolus wöchentlich } \\
\text { rhuMab VEGF } 10 \mathrm{mg} / \mathrm{kg} \text {, q2w }\end{array}$} & 13,8 & 17 & 5,2 \\
\hline & 35 & & & $>17,3$ & 40 & 9,0 \\
\hline & 33 & Phase II & & 16,1 & 24 & 7,2 \\
\hline \multirow{2}{*}{$\begin{array}{l}\text { Hurwitz et al. } \\
{[22]}\end{array}$} & 412 & \multirow{2}{*}{$\begin{array}{l}\text { Erstlinien- } \\
\text { therapie } \\
\text { randomisiert/ } \\
\text { Phase III }\end{array}$} & IFL + Placebo & $15,6^{* *}$ & $35^{* *}$ & $6,2^{*}$ \\
\hline & 403 & & IFL + Bevacizumab & $20,3^{* *}$ & $45^{* *}$ & $10,8^{*}$ \\
\hline
\end{tabular}

PFS: Medianes progressionsfreies Überleben, FU/FA: 5-FU/Leukovorin,IFL: Irinotecan/5-FU/Leukovorin. ${ }^{*} \mathrm{p}<0,003 ;{ }^{* *} \mathrm{p}<0,001$ lag die mediane Überlebenszeit bei über 20 Monaten. Dies ist wahrscheinlich sowohl auf die Erstlinien- als auch auf die Zweitlinientherapie zurückzuführen (Abb. 1).

Aufgrund dieser Daten sollte heute durchweg eine Erstlinientherapie mit 5-FU-Dauerinfusion und Irinotecan bzw. Oxaliplatin bei den Patienten durchgeführt werden. Aufgrund der beiden Studien, die einen Überlebensvorteil für die Irinotecan-haltige Erstlinientherapie zeigten, liegen hier gewisse Vorteile im Erstlinieneinsatz von Irinotecan. Sollten die Patienten nicht mit einer Kombinationstherapie behandelt werden, ist dies zu begründen, da zu befürchten ist, dass dies für den Patienten ein nachteiliger Therapieansatz ist.

\section{Orale Fluoropyrimidine in Kombination mit Irinotecan oder Oxaliplatin}

Die oralen Fluoropyrimidine haben das Potenzial, die 5-FUDauerinfusion zu ersetzen, sodass dem Patienten kein Port zu implantieren ist bzw. keine Pumpentherapie notwendig scheint. In Phase II-Studien zeigen sich sehr viel versprechen- de Ergebnisse für Capecitabin mit Irinotecan (Tab. 5) sowie für Capecitabin mit Oxaliplatin. Auch die Toxizität beider Behandlungsregime scheint günstig zu sein, sodass der randomisierte Vergleich zwischen 5-FU-Dauerinfusion mit Folinsäure und einem Kombinationspartner Irinotecan und Oxaliplatin sinnvoll ist. Diese Studien (z.B. EORTC 40015) laufen in Deutschland und europaweit und werden den Stellenwert der oralen Fluoropyrimidine in der Kombinationsbehandlung besser klären (Abb. 2).

\section{Neue Therapieansätze}

Der epidermale Wachstumsfaktorrezeptor (EGF-Rezeptor) ist bei etwa $80 \%$ der Patienten mit kolorektalem Karzinom auf den Tumorzellen exprimiert [19]. Der klinisch am weitesten entwickelte Antikörper gegen den EGF-Rezeptor ist Cetuximab, welcher in einer ersten randomisierten Studie bei Irinotecan-vorbehandelten Patienten geprüft wurde (Tab. 6). Hier erhielten die Patienten entweder Cetuximab allein oder Cetuximab in Kombination mit Irinotecan in einer in der Vor- 
therapie tolerablen Dosis [20]. Patienten, die mit der Kombination behandelt wurden, hatten eine signifikant höhere Remissionsrate (23 versus $11 \%, \mathrm{p}=0,007$ ). Auch die Rate an Tumorstabilisierungen der zuvor tumorprogredienten Patienten war signifikant verbessert worden (55 versus $32 \%, \mathrm{p}=0,0001$ ). Die mediane progressionsfreie Zeit war ebenfalls mit 4,1 versus 1,5 Monaten signifikant ( $\mathrm{p}<0,0001)$ länger. Damit ist Cetuximab eines der wirksamsten Medikamente in der Behandlung von Patienten des metastasierten kolorektalen Karzinoms und scheint in der Lage zu sein, eine Irinotecanresistenz zu durchbrechen. Es ist zu erwarten, dass der Einsatz von Cetuximab in der Erstlinientherapie eine wesentliche weitere Verbesserung bringen wird [21]. Interessanterweise war die Wirksamkeit von Cetuximab unabhängig davon, in welchem Ausmaß die Patienten den EGF-Rezeptor exprimierten, korrelierte aber mit der Schwere der Hautreaktionen, die durch Cetuximab induziert wurde. Patienten mit höhergradigen, akneartigen Hautveränderungen hatten eine höhere Wahrscheinlichkeit, auf eine Cetuximab-haltige Therapie anzusprechen und länger zu überleben.

Ein weiterer Fortschritt könnte die Beeinflussung des vaskulären endothelialen Wachstumsfaktors (VEGF) bzw. seines Rezeptors sein. Der Antikörper Bevacizumab ist gegen im Blut zirkulierendes VEGF gerichtet und verhindert so die Angiogenese von Tumorzellen. Im Rahmen einer randomisierten Studie [22] an über 800 Patienten war Bevacizumab in der Lage, eine IFL (Irinotecan/5-FU/Leukovorin)-haltige Chemotherapie bezüglich der Remissionsrate, der Zeit bis zur Tumorprogression sowie auch der medianen Überlebenszeit signifikant zu verbessern (Tab. 7). Bevacizumab gehört damit sicherlich zu den weiteren neuen sehr viel versprechenden Me- dikamenten. Weder Cetuximab noch Bevacizumab sind jedoch zur Zeit zugelassen.

In präklinischen Modellen konnte der antiproliferative und proapoptotische Effekt von Cyclooxygenase-2 (COX-2)-Inhibitoren gezeigt werden [23]. Insbesondere haben COX-2-Inhibitoren einen Stellenwert in der Unterdrückung von Kolonpolypen. Darüber hinaus besteht ein Synergismus mit Chemotherapie [24]. In ersten klinischen Erfahrungen konnte die Durchführbarkeit einer Chemotherapie in Kombination mit Celecoxib gezeigt werden. Die Daten legen nahe, dass Celecoxib die Rate an Nebenwirkungen von Capecitabin senkt und möglicherweise die antineoplastische Aktivität steigert [25]. Die EORTC und andere Studiengruppen untersuchen daher zur Zeit den Stellenwert von Celecoxib in Kombination mit einer Chemotherapie im Rahmen der EORTC Studie 40015.

\section{Fazit}

Aufgrund der vorliegenden Daten sollten Patienten unbedingt mit einer Kombinationschemotherapie behandelt werden. Ein sequentielles Vorgehen mit 5-FU-Monotherapie oder oralen Fluoropyrimidenen gefolgt von Irinotecan-haltigen oder Oxaliplatin-haltigen Kombinationen erscheint nach der derzeitigen Datenlage das schlechtere Vorgehen zu sein. Die hohen medianen Überlebensraten von über 20 Monaten sind ausnahmslos in Studien beobachtet worden, bei denen eine kombinierte Erstlinientherapie eingesetzt wurde, und die Patienten die Möglichkeit hatten, später das alternative Medikament $\mathrm{zu}$ erhalten. Es ist zu erwarten, dass die neuen Antikörper einen weiteren Fortschritt der Therapie bewirken.

\section{Literatur}

1 Köhne CH, Kretzschmar A, Wils J: First-Line chemotherapy for colorectal carcinoma - we are making progress. Onkologie 1998;21:280-289.

2 Piedbois P, Michelies S: Survival benefit of 5FU/LV over $5 \mathrm{FU}$ bolus in patients with advanced colorectal cancer: An updated meta-analysis based on 2,751 patients. Proc American Soc Clin Oncol 2003; 22:156

3 Efficacy of intravenous continuous infusion of fluorouracil compared with bolus administration in advanced colorectal cancer. Meta-analysis Group In Cancer. J Clin Oncol 1998;16:301-308.

4 Van Cutsem E, Twelves C, Cassidy J, Allman D, Bajetta E, Boyer M, Bugat R, Findlay M, Frings S, Jahn M, McKendrick J, Osterwalder B, PerezManga G, Rosso R, Rougier P, Schmiegel WH, Seitz JF, Thompson P, Vieitez JM, Weitzel C, Harper P; Xeloda Colorectal Cancer Study Group: Oral capecitabine compared with intravenous fluorouracil plus leucovorin in patients with metastatic colorectal cancer: Results of a large phase III study. J Clin Oncol 2001;19:4097-4106.
5 Hoff PM, Ansari R, Batist G, Cox J, Kocha W, Kuperminc M, Maroun J, Walde D, Weaver C, Harrison $\mathrm{E}$, Burger HU, Osterwalder B, Wong AO, Wong R: Comparison of oral capecitabine versus intravenous fluorouracil plus leucovorin as firstline treatment in 605 patients with metastatic colorectal cancer: Results of a randomized phase III study. J Clin Oncol 2001;19:2282-2292.

6 Carmichael J, Popiela T, Radstone D, Falk S, Borner M, Oza A, Skovsgaard T, Munier S, Martin C: Randomized comparative study of tegafur/uracil and oral leucovorin versus parenteral fluorouracil and leucovorin in patients with previously untreated metastatic colorectal cancer. J Clin Oncol 2002; 20:3617-3627.

7 Douillard JY, Hoff PM, Skillings JR, Eisenberg P, Davidson N, Harper P, Vincent MD, Lembersky BC, Thompson S, Maniero A, Benner SE: Multicenter phase III study of uracil/tegafur and oral leucovorin versus fluorouracil and leucovorin in patients with previously untreated metastatic colorectal cancer. J Clin Oncol 2002;20:3605-3616.
8 Rougier P, Van Cutsem E, Bajetta E, Niederle N, Possinger K, Labianca R, Navarro M, Morant R, Bleiberg H, Wils J, Awad L, Herait P, Jacques C: Randomised trial of irinotecan versus fluorouracil by continuous infusion after fluorouracil failure in patients with metastatic colorectal cancer. Lancet 1998;352:1407-1412.

9 Douillard JY, Cunningham D, Roth AD, Navarro M, James RD, Karasek P, Jandik P, Iveson T, Carmichael J, Alakl M, Gruia G, Awad L, Rougier P: Irinotecan combined with fluorouracil compared with fluorouracil alone as first-line treatment for metastatic colorectal cancer: A multicentre randomised trial. Lancet 2000;355:1041-1047.

10 Saltz LB, Cox JV, Blanke C, Rosen LS, Fehrenbacher L, Moore MJ, Maroun JA, Ackland SP, Locker PK, Pirotta N, Elfring GL, Miller LL: Irinotecan plus fluorouracil and leucovorin for metastatic colorectal cancer. Irinotecan Study Group. N Engl J Med 2000;343:905-914.

11 Köhne CH, Van Cutsem E, Wils J, Bokemeyer C, el-Safadi M, Lutz MP, et al.: Irinotecan improves the activity of the AIO regimen in metastatic colorectal cancer: Results of EORTC GI Group study 40986. Proc ASCO 2003;22:154. 
12 Giacchetti S, Perpoint B, Zidani R, Le Bail N, Faggiuolo R, Focan C, Chollet P, Llory JF, Letourneau Y, Coudert B, Bertheaut-Cvitkovic F, LarregainFournier D, Le Rol A, Walter S, Adam R, Misse JL, Levi F: Phase III multicenter randomized trial of oxaliplatin added to chronomodulated fluorouracil-leucovorin as first-line treatment of metastatic colorectal cancer. J Clin Oncol 2000;18: 136-147.

13 de Gramont A, Figer A, Seymour M, Homerin M, Hmissi A, Cassidy J, Boni C, Cortes-Funes H, Cervantes A, Freyer G, Papamichael D, Le Bail N, Louvet C, Hendler D, de Braud F, Wilson C, Mor van F, Bonetti A: Leucovorin and Fluorouracil with or without Oxaliplatin as first-line treatment in advanced colorectal cancer. J Clin Oncol 2000;18: 2938-2947.

14 Grothey A, Deschler B, Kroening H, Ridwelski K, Reichardt P, Kretzschmar A, et al.: Phase III study of bolus 5-fluorouracil (5-FU)/ folinic acid (FA) (Mayo) vs weekly high-dose 24h 5-FU infusion/ FA + oxaliplatin (OXA) (FUFOX) in advanced colorectal cancer (ACRC). Proc Am Soc Clin Oncol 2002;21:156

15 Rothenberg ML, Oza AM, Bigelow RH, Berlin JD, Marshall JL, Ramanathan RK, Hart LL, Gupta S, Garay CA, Burger BG, Le Bail N, Haller DG: Superiority of oxaliplatin and fluorouracil-leucovorin compared with either therapy alone in patient with progressive colorectal cancer after irinotecan and fluorouracil-leucovorin: interim results of a phase III trial. J Clin Oncol 2003;21:2059-2069.

16 Goldberg RM, Morton RF, Sargent D, Fuchs CS, Ramanathan RK, Williamson SK, et al.: N9741: oxaliplatin (Oxal) or CPT-11 + 5-Fluororuacil $(5 \mathrm{FU}) /$ leucovorin $(\mathrm{LV})$ or oxal+CPT-11 in advanced colorectal cancer (CRC). Initial toxicity and response data from a GI intergroup study. Proc ASCO 2002;18:305.

17 Tournigand C, Louvet C, Quinaux E, Andre T, Lledo $\mathrm{G}$, Flesh $\mathrm{M}$ et al. FOLFIRI followed by FOLFOX versus FOLFOX followed by FOLFIR in metastatic colorectal cancer (MCRC): Final results of a phase III study. Proc ASCO 2001;20:494.

18 Köhne CH, Wils J, Lorenz M, Schöffski P, Voigtmann R, Bokemeyer C, et al. Randomized phase III study of high dose 5-Fluorouracil given as a weekly 24 hour infusion with or without leucovorin vs. bolus 5-Fluorouracil plus leucovorin in advanced colorectal cancer. EORTC Gastrointestinal Group Study 40952. J Clin Oncol 2003;in press.

19 McKay JA, Murray LJ, Curran S, Ross VG, Clark C, Murray GI, Cassidy J, McLeod HL: Evaluation of the epidermal growth factor receptor (EGFR) in colorectal tumours and lymph node metastases. Eur J Cancer 2002;38:2258-2264.

20 Cunningham D, Humblet Y, Siena D, Khayat D, Bleiberg H, Santoro A, et al.: Cetuximab (C225) alone or in combination with irinotecan (CPT-11) in patients with epidermal growth factor receptor (EGFR)-positive, irinotecan-refractory metastatic colorectal cancer (MCRC). Proc ASCO 2003;22: 256.
21 Schoeffski P, Lutz MP, Folprecht G, Beutel G, Seufferlein T, Marschner JP, et al.: Cetuximab (C225) plus irinotecan (CPT-11) plus infusional 5FU-folinic acid (FA) is safe and active in metastatic colorectal cancer (MCRC), that expresses epidermal growth factor receptor (EGFR). Proc ASCO 2002; 633.

22 Hurwitz H, Fehrenbacher L, Cartwright T, Hainsworth J, Heim W, Berlin J, et al.: Bevacizumab (a monoclonal antibody to vascular endothelial growth factor) prolongs survival in first-line colorectal cancer (CRC): Results of a phase III trial of bevacizumab in combination with bolus IFL (irinotecan, 5-fluorouracil, leucovorin) as first-line therapy in subjects with metastatic CRC. Proc ASCO 2003;22:254.

23 Masferrer JL, Leahy KM, Koki AT, Zweifel BS, Settle SL, Woerner BM, DA, Flickinger AG, Moore RJ, Seibert K: Antiangiogenic and antitumor activities of cyclooxygenase-2 inhibitors. Cancer Res 2000;60:1306-1311.

24 Trifan OC, Durham WF, Salazar VS, Horton J, Levine BD, Zweifel BS, Davis TW, Masferrer JL: Cyclooxygenase-2 inhibition with celecoxib enhances antitumor efficacy and reduces diarrhea side effect of CPT-11. Cancer Res 2002;62:57785784.

25 Lin E, Morris J, Chau N, Crane C, Wolff R, Janjan $\mathrm{N}$, et al.: Celecoxib attenuated capecitabine induced hand-and-foot syndrome (HFS) and diarrhea and improved time to tumor progression in metastatic colorectal cancer (MCRC). Proc ASCO 2003;21:176a.

26 Kerr DJ, ten Bokkel Huinink WW, Ferry D, Rea DW, Boussard BM, Oulid-Aissa D, et al.: A phase I/II study of CPT-11 in combination with capecitabine as first line chemotherapy for metastatic colorectal cancer (MCRC). Proc ASCO 2002; 643:2002.

27 Patt Y, Lin E, Leibann J, Miller W, Lee F, Keiser W, et al.: Capecitabine plus irinotecan for chemotherapy-naïve patients with metastatic colorectal cancer (MCRC): US multicenter phase II trial. Proc ASCO 2003;21:176

28 Munoz A, Salud A, Escudero P, Alonso V, Sanz J, et al.: Irinotecan (CPT-11) and capecitabine (C) as first line treatment of locally advanced or metastatic colorectal cancer (CRC). Proc ASCO 2003;22: 176

29 Dickson N, Burris HA, Foulke R, Schwartzberg LS, Kalman L, Bertoli L, et al.: Flat-dose continuous capecitabine with irinotecan (CPT) in 1st line advanced colorectal cancer (ACRC): Early results of a phase II trial. Proc ASCO 2003;22:176.

30 Bollina R, Beretta G, Toniolo D, Cozzi C, Belloni P, Clerici M, et al.: Capecitabine (C) and irinotecan (i) (CAPIRI): A good combination in elderly patients (pts) with advanced colorectal cancer (ACRC) as first-line chemotherapy (CT). Preliminary results of a phase II trial. Proc ASCO 2003; 22:175.
31 Schleucher N, Tewes M, Achterrath W, Hense J, Omidi S, Frings S, et al.: Extended Phase I Study of Capecitabine in Combination with a Weekly Schedule of Irinotecan as First-Line Chemotherapy in Metastatic Colorectal Cancer. Proc ASCO 2001;20: $141 \mathrm{a}$

32 Grothey A, Jordan K, Kellner O, Constantin C, Dietrich G, Kroening H, et al.: Randomized phase I trial of capecitabine plus irinotecan vs. capecitabine plus oxaliplatin as first-line therapy of advanced colorectal cancer (ACRC). Ann Oncol 2002;13(S5): 71

33 Cassata A, Stani SC, Alu M, Procopio G, Cortinovis $\mathrm{D}$, Beretta $\mathrm{E}$, et al.: Ongoing phase II trial with two schedules of irinotecan (CPT-11) in combination with capecitabine as first-line chemotherapy in patients with advanced colorectal cancer (ACRC) Proc ASCO 2001;20:573

34 Park YS, Chun JH, Lee HG, Yoon SM, Shin E, Kim EE, et al.: A phase II trial of irinotecan and capecitabine in patients with advanced colorectal cancer (ACRC). Proc ASCO 22;176:2003.

35 Gold PJ, Godfrey T, Dhami M, Andria M, Fischer D, et al.: Capecitabine and irinotecan as 1st line therapy for patients with metastatic colorectal cancer: Phase II study preliminary results. Proc ASCO 2003;22:178

36 Borner M, Dietrich D, Popescu R, Wernli M, Saletti $P$, Rauch D, et al.: A randomized phase II trial of capecitabine (CAP) and two different schedules of irinotecan (IRI) in first-line treatment of metastatic colorectal cancer (MCC). Proc ASCO 2003;22:178.

37 Saltz L, Rubin M, Hochster H, Tchemeydian NS, Waksal HW, Needle M, et al.: Cetuximab (IMCC225) Plus Irinotecan (CPT-11) is Active in CPT11-Refractory Colorectal Cancer (CRC) that Expresses Epidermal Growth Factor Receptor (EGFR). Proc ASCO 2001;20:3a.

38 Saltz L, Meropol NJ, Loehrer PJ, Waksal HW, Needle M, Mayer RJ: Single agent IMC-C225 (Erbitux) has activity in CPT-11- refractory colorectal cance (CRC) that expresses the epidermal growth factor receptor (EGFR). Proc ASCO 2002;21:127a.

39 Lutz MP, Schöffski P, Folprecht G, Haag C, Seufferlein T, Beutel G, et al.: A phase I/II study of cetuximab (C225) plus irinotecan (CPT-11) and 24h infusional 5FU/folinic acid (FA) in the treatment of metastatic colorectal cancer (MCRC) expressing the epidermal growth factor receptor (EGFR). Ann Oncol 2002;13(S5):73.

40 Rosenberg AH, Loehrer PJ, Needle M, Waksal HW, Hollywood E, Ramos L, et al.: Erbitux (IMCC225) plus weekly irinotecan (CPT-11), fluorouracil (5FU) and leucovorin (LV) in colorectal cancer (CRC) that expresses the epidermal growth factor receptor (EGFr). Proc ASCO 2002;21:135a.

41 Bergsland E, Hurwitz H, Fehrenbacher L, Meropo NJ, Novotny L, Gaudreault J, et al.: A randomized phase II trial comparing rhuMAb VEGF (recombinant humanized monoclonal antibody to vascular endothelial cell growth factor) plus 5-fluorouracil/ leucovorin (FU/LV) to FU/LV alone in patient with metastatic colorectal cancer. Proc ASCO 2000;19:939 